\title{
Value of artificial intelligence model based on unenhanced computed tomography of urinary tract for preoperative prediction of calcium oxalate monohydrate stones in vivo
}

\author{
Lei Tang ${ }^{1,2 \#}$, Wuchao $\mathrm{Li}^{2 \#}$, Xianchun Zeng ${ }^{2} \wedge$, Rongpin Wang ${ }^{2}$, Xiushu Yang ${ }^{3} \wedge$, Guangheng Luo ${ }^{3}$, \\ Qijian Chen ${ }^{4}$, Lihui Wang ${ }^{4}$, Bin Song ${ }^{1 \wedge}$ \\ ${ }^{1}$ Department of Radiology, West China Hospital, Sichuan University, Chengdu, China; ${ }^{2}$ Department of Radiology, Guizhou Provincial People's \\ Hospital, Guiyang, China; ${ }^{3}$ Department of Urological Surgery, Guizhou Provincial People's Hospital, Guiyang, China; ${ }^{4}$ College of Computer \\ Science and Technology, Guizhou University, Guiyang, China \\ Contributions: (I) Conception and design: B Song, R Wang; (II) Administrative support: B Song, G Luo; (III) Provision of study materials or patients: \\ X Yang, G Luo; (IV) Collection and assembly of data: X Zeng, W Li, X Yang; (V) Data analysis and interpretation: Q Chen, L Wang, L Tang; (VI) \\ Manuscript writing: All authors; (VII) Final approval of manuscript: All authors. \\ "These authors contributed equally to this work. \\ Correspondence to: Bin Song. Department of Radiology, West China Hospital, Sichuan University, No. 37 Guoxue Alley, Wuhou District, Chengdu \\ 610041, China. Email: songlab_radiology@163.com.
}

\begin{abstract}
Background: Urolithiasis is a global disease with a high incidence and recurrence rate, and stone composition is closely related to the choice of treatment and preventive measures. Calcium oxalate monohydrate (COM) is the most common in clinical practice, which is hard and difficult to fragment. Preoperative identification of its components and selection of effective surgical methods can reduce the risk of patients having a second operation. Methods that can be used for stone composition analysis include infrared spectroscopy, X-ray diffraction, and polarized light microscopy, but they are all performed on stone specimens in vitro after surgery. This study aimed to design and develop an artificial intelligence (AI) model based on unenhanced computed tomography (CT) images of the urinary tract, and to investigate the predictive ability of the model for COM stones in vivo preoperatively, so as to provide surgeons with more accurate diagnostic information.
\end{abstract}

Methods: Preoperative unenhanced CT images of patients with urinary calculi whose components were determined by infrared spectroscopy in a single center were retrospectively analyzed, including 337 cases of COM stones and 170 of non-COM stones. All images were manually segmented and the image features were extracted, and randomly divided into the training and testing sets in a ratio of 7:3. The least absolute shrinkage and selection operation algorithm (LASSO) was used to construct the AI model, and classification of the training and testing sets was carried out.

Results: A total of 1,218 radiomics imaging features were extracted, and 8 features with non-zero coefficients were finally obtained. The sensitivity, specificity and accuracy of the AI model were $90.5 \%$, $84.3 \%$ and $88.5 \%$ for the training set, and $90.1 \%, 84.3 \%$ and $88.3 \%$ for the testing set. The area under the curve was 0.935 for the training set and 0.933 for the testing set.

Conclusions: The AI model based on unenhanced CT images of the urinary tract can predict COM and non-COM stones in vivo preoperatively, and the model has high sensitivity, specificity and accuracy.

Keywords: Calcium oxalate monohydrate (COM); component analysis; artificial intelligence (AI); unenhanced CT

\footnotetext{
^, Lei Tang, 0000-0003-1582-1023; Xianchun Zeng, 0000-0003-0857-3834; Rongpin Wang, 0000-0001-7587-4181; Xiushu Yang, 00000001-9423-0648; Bin Song, 0000-0002-7269-2101.
} 
Submitted Mar 01, 2021. Accepted for publication Jun 03, 2021.

doi: 10.21037/atm-21-965

View this article at: https://dx.doi.org/10.21037/atm-21-965

\section{Introduction}

Urolithiasis is a global disease with a high incidence and recurrence rate, with a worldwide prevalence of $2-20 \%$ (1), a 1 -year recurrence rate of $6-17 \%$, and a 5 -year recurrence rate of $21-53 \%$ (2). The main clinical manifestations are acute renal colic, hematuria, and urinary tract irritation signs (frequent micturition, urgency, and dysuria). Severe cases may be combined with long-term, chronic obstructive hydronephrosis, eventually causing varying degrees of kidney function damage, and even endangering the lives of patients. There are many types of urinary stones, and the pathogenesis involves the combined effects of abnormal human metabolism, urinary tract obstruction, infection, drugs, and a variety of internal and external environmental factors (such as age, gender, race, genetics, geography, climate, water intake, and dietary habits) (3-5). Calculi composition is closely related to the choice of treatment and preventive measures. For larger stones that are difficult to break up, the results of extracorporeal shock wave lithotripsy (ESWL) are often unsatisfactory, and flexible ureteroscopic lithotripsy or percutaneous nephrolithotripsy (PCNL) is required.

There are various types of urinary calculi, and calcium oxalate (CO) calculi are the most common clinically, accounting for $80 \%$ of all stones (6). CO crystals are available in three hydrated forms: heat-stable calcium oxalate monohydrate (COM), substable calcium oxalate dihydrate (COD), and unstable calcium oxalate trihydrate (COT); the former two of which can be the main components of urinary stones and COM is the most common, while the latter COT has never been found in urine or stone crystals (7). In terms of crystal properties, most COM stones are brown, mulberry-shaped, hard and not easy to be dusted; COD stones are mostly pale yellowbrown, with needle-like protrusions visible on the surface, brittle, and easy to be broken. COM stones are the most common and difficult to fragment in clinical practice, and it is of clinical significance to clarify their chemical composition before surgery to facilitate the correct choice of surgery.

The methods that can be used for stone composition analysis include infrared spectroscopy (IRS), X-ray diffraction (XRD), thermal analysis, polarized light microscopy, emission spectroscopy, and chemical titration reaction; of which, the most commonly used is IRS (8). However, these methods can only be performed in vitro, and stone specimens must be obtained by surgery, or after being passed out of the body. How to preoperatively clarify the composition of stones in the body in a simple, fast and noninvasive way is important for the diagnosis, treatment, prognosis and prevention of recurrence of urolithiasis, and has always been the focus of urologists.

Artificial intelligence (AI) combined with imaging, using advanced computer algorithms to deeply mine the multidimensional features embedded in medical imaging data, has been widely used in various fields of radiology, showing potential and broad application prospects in disease diagnosis, lesion segmentation, tumor grading and staging, and prognostic prediction. Its accuracy in classification and grading of many diseases, including pneumothorax, pulmonary nodules, and renal cell carcinoma and others, is almost comparable to and even surpasses clinicians (9-12).

In this study, we retrospectively collected urolithiasis cases whose composition was determined by IRS, aiming to develop an AI model based on preoperative unenhanced urinary tract CT for automatic prediction of stone composition, in order to provide urologists with detailed stone diagnostic information for further guidance in the selection of treatment and surgical modality. We present the following article in accordance with the TRIPOD reporting checklist (available at https://dx.doi. org/10.21037/atm-21-965).

\section{Methods}

\section{Data set}

This retrospective study was conducted in accordance with the Declaration of Helsinki (as revised in 2013) and approved by institutional ethics board of Guizhou Provincial People's Hospital (No. [2020]184), and individual consent was waived. The data set consisted of CT imaging data of urolithiasis patients treated at Guizhou Provincial People's Hospital. Cases with postoperative IRS stone composition analysis (LIIR-20, Lamoride Company, Tianjin, China) and preoperative CT scan data of the urinary tract in our hospital from January 2015 to December 2019 were 


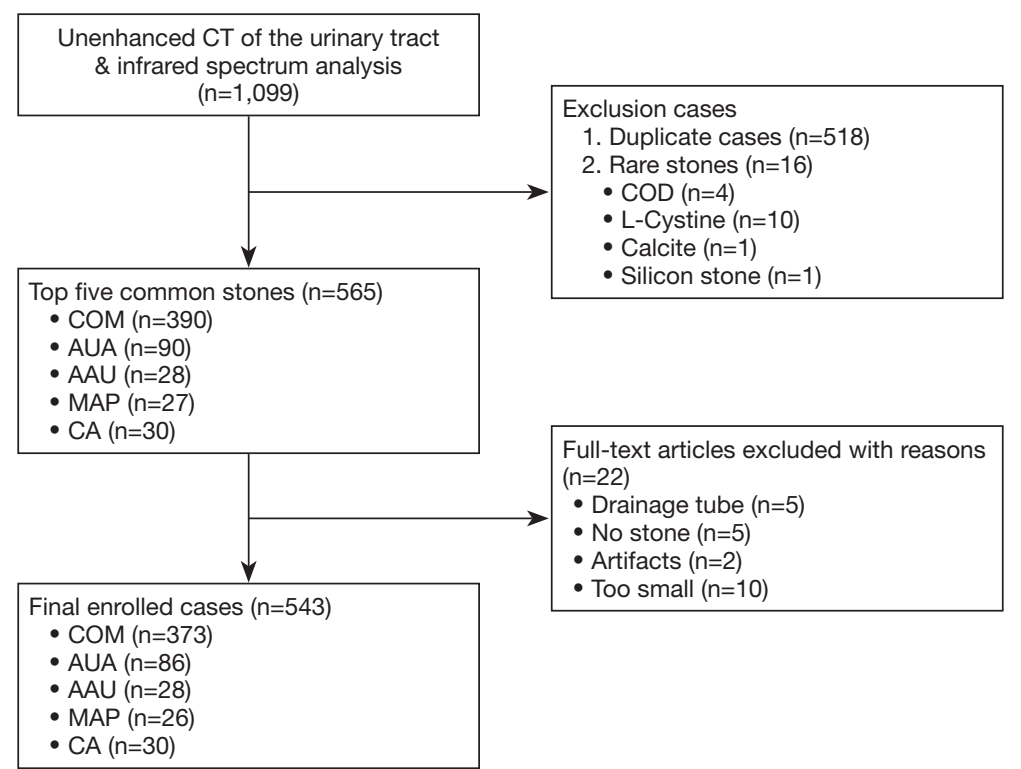

Figure 1 Case screening flow chart. COM, calcium oxalate monohydrate; COD, calcium oxalate dihydrate; AUA, anhydrous uric acid; AAU, ammonium urate; MAP, magnesium ammonium phosphate; CA, carbonate apatite.

retrospectively collected. Mixed stones are more common clinically than pure stones; there is no uniform classification standard (13), and the treatment of mixed stones is selected based on the major component (14-16). Therefore, we named the collected stones by their main component. The most harmful stones are often treated first in clinical practice; thus, for a particular patient with multiple stones, we defined all stones in the body as one sample, named after the main component of the surgically obtained specimen.

Exclusion criteria were: (I) duplicate cases (multiple stone samples submitted by the same patient at the same time, with multiple IRS records); (II) nephrostomy tube or ureteral drainage tube placement and overlapping with the calculi (which may affect stone segmentation and feature extraction); (III) no stones on CT images (calculi had passed spontaneously before CT examination in some patients); (IV) obvious respiratory or motion artifacts; (V) stones too small: diameter $<3 \mathrm{~mm}$. The patient's age and gender, and stone recurrence were recorded according to the clinical history; stone site, number of stones, and stone morphology (staghorn or nonstaghorn) were recorded according to CT images; and the chemical composition of the stones was recorded according to IRS analysis.

A total of 543 patients were finally collected, and the five most common stones were: COM, anhydrous uric acid (AUA), carbonate apatite (CA), ammonium urate (AAU), and magnesium ammonium phosphate (MAP). Among them, there were 373 cases of COM calculi and 170 of non-COM calculi. The case screening process is shown in Figure 1.

\section{CT equipment and parameters}

The scans ranged from the upper edge of the kidney to the lower edge of the sciatic tuberosity and were performed with three different models of CT scanners from Siemens, Germany: (I) SOMATOM Definition CT: Real-time exposure dose auto-adjustment (CARE Dose 4D and CARE $\mathrm{kV}$ on), reference tube voltage $120 \mathrm{kV}$, reference tube current $210 \mathrm{mAs}$, detector width $64 \mathrm{~mm} \times 0.6 \mathrm{~mm}$, pitch 1.0; filtered inverse projection (FBP) reconstruction, convolution function $\mathrm{Bf} 40$, layer thickness $1.0 \mathrm{~mm}$. (II) SOMATOM Definition AS+: Real-time exposure dose autoadjustment, reference tube voltage $120 \mathrm{kV}$, reference tube current $210 \mathrm{mAs}$, detector width $64 \mathrm{~mm} \times 0.6 \mathrm{~mm}$, pitch 1.2; FBP reconstruction, Bf 40, layer thickness $1.0 \mathrm{~mm}$. (III) Somatom Force CT: CARE Dose 4D and CARE kV on, reference tube voltage $100 \mathrm{kV}$, reference tube current $180 \mathrm{mAs}$, detector width $96 \mathrm{~mm} \times 0.6 \mathrm{~mm}$, pitch 1.2 ; ADMIRE reconstruction (intensity level 3), convolution function $\mathrm{Br}$ 40, layer thickness $1.0 \mathrm{~mm}$.

\section{CT image acquisition and stone segmentation}

Thin-layer $(1.0 \mathrm{~mm})$, axial, unenhanced urological CT 


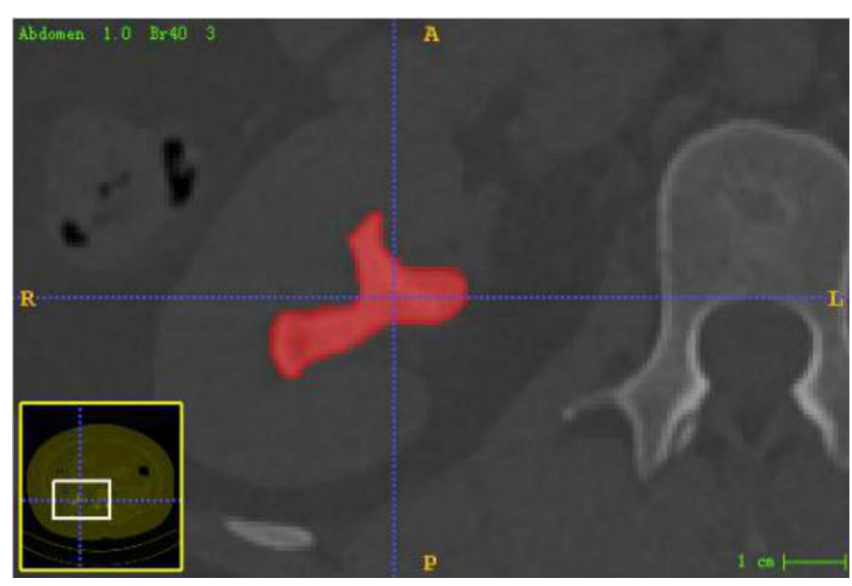

Figure 2 Manual segmentation interface for urinary stones (ITKSNAP software).

images of the enrolled cases were exported in DICOM format from the Picture Archiving and Communication System (PACS). A radiologist with $>10$ years of diagnostic experience imported the CT images one by one into the 3D image segmentation software package ITK-SNAP (Version 3.8.0-Beta, University of Pennsylvania), drew the regions of interest (ROI) layer by layer, and saved the segmented images in nii.gz format (Figure 2). ITK-SNAP software comes with image registration function, which can automatically adjust the image gray level, that is, the standardization of the image. Stone segmentation principle: drawing on the axial image; ROI is delineated along the stone edge, trying to include the stone as a whole and avoiding the inclusion of surrounding renal tissue, blood vessels, fat or image artifacts to ensure data reliability.

\section{Extraction and screening of stone features}

According to the AI standardization process, data cleaning or standardization should be carried out before ROI feature extraction, which aims to eliminate data differences caused by different devices and scan parameters and reduce data errors. PyRadiomics software (Version 3.0, http:// pyradiomics.readthedocs.io/) was used to normalize the values of all ROIs, to adjust the scattered pixel values on each layer of images to a nearly uniform standard. Then, PyRadiomics software was used to extract the stone features. A total of 1,218 radiomics features were extracted, including six types of feature parameters: first-order features $(n=18)$; morphological features $(n=14)$; gray level co-occurrence matrix $(\mathrm{GLCM})$ features $(\mathrm{n}=22)$; grayscale correlation matrix (GLDM) features ( $\mathrm{n}=14)$; grayscale size region matrix (GLSZM) features $(\mathrm{n}=16)$; gray running length matrix (GLRLM) features ( $\mathrm{n}=16)$.

The intraclass and interclass correlation coefficient (ICC) was used to evaluate the consistency of the extracted features, and features with ICC $>0.75$ were considered to be consistent, reproducible and stable (17). In this step, a total of 8 features with ICC $\leq 0.75$ were deleted, leaving 1,210 features. After that, the least absolute shrinkage and selection operator (LASSO) algorithm was used to screen the stone feature parameters.

\section{AI model construction and testing}

The sampling software package of $\mathrm{R}$ (version 3.6.3) was used to randomly allocate the enrolled patients into training and testing sets at a ratio of 7:3. The characteristic parameters obtained after dimension reduction were used to construct the binary LASSO model. Seventy percent of the samples were used as the training set $(\mathrm{n}=381)$ for machine learning and training of the model, and $30 \%$ of the samples were used as the testing set $(n=162)$ to test the diagnostic efficiency of the model. The predictive effectiveness of the AI model was evaluated in the training and testing set respectively; the accuracy, sensitivity and specificity of the model were calculated based on the optimal cut-off value of the training set; and the ROC curve and area under the curve (AUC) were obtained. The steps of AI model construction and analysis are shown in Figure 3.

\section{Statistical analysis}

Statistical analysis of the clinical characteristics of the enrolled cases was performed using SPSS software (version 22.0, IBM Corporation). The chi-square test was used to compare the difference of stone composition among different genders, occurrence sites, occurrence numbers (single/multiple), and different stone shapes, and $\mathrm{P}<0.05$ was statistically significant.

\section{Results}

\section{Clinical and CT findings}

Of the 543 patients, 373 were in the COM group and 170 in the non-COM group (including $86 \mathrm{AUA}, 28 \mathrm{AAU}, 26 \mathrm{MAP}$, and $30 \mathrm{CA}$ stones). There were 186 cases of pure calculi (142 COM, 38 AUA, and $6 \mathrm{AAU}$ ), and 357 cases were 


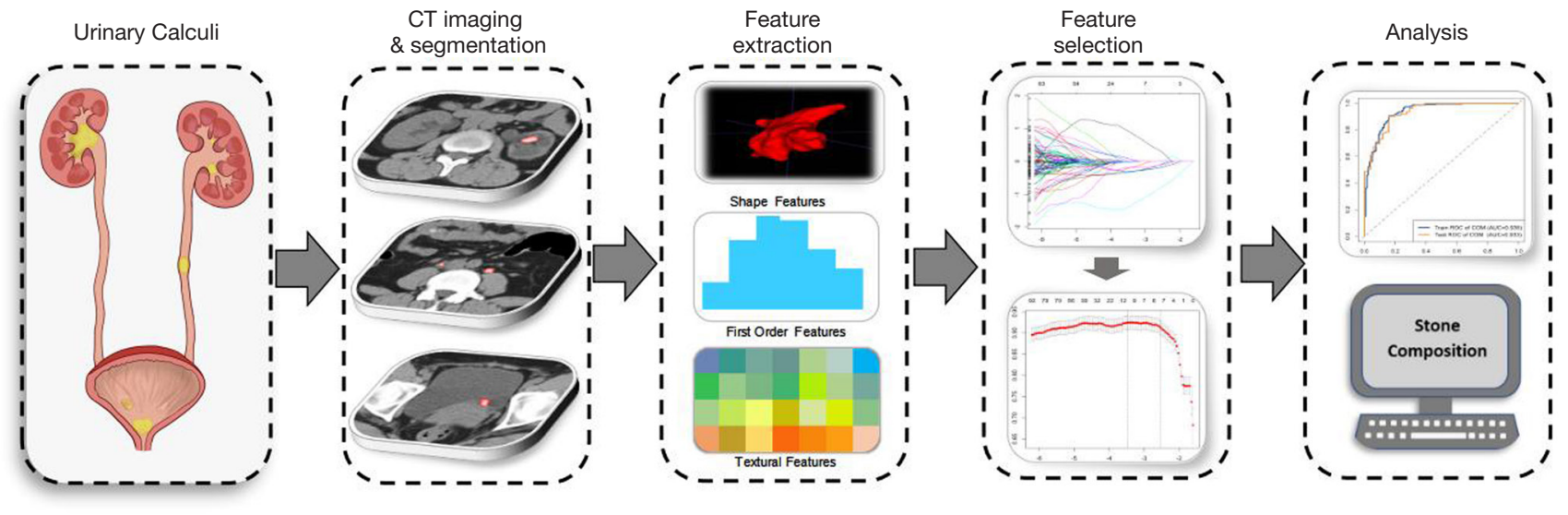

Figure 3 Artificial intelligence analysis step-by-step diagram.

mixed calculi; among which, MAP and CA were all mixed. In the COM group, there were 275 men and 98 women; age $45.55 \pm 16.14$ years, including 23 aged $<18$ years and 350 $>18$ years; 7 cases of recurrence after 1 year and 3 after 2 years; 1 case of combined horseshoe kidney malformation; 160 cases of single and 213 cases of multiple stones; 161 cases on the left, 127 cases on the right, and 73 bilateral cases. There were 177 cases of kidney stones, 92 ureteral stones, 12 bladder stones, 88 kidney-ureteral stones, 4 kidney-bladder stones, 24 staghorn stones, and 349 nonstaghorn stones (Table 1).

\section{Selected stone features}

The 1,210 features were screened by 10 -fold cross validation, and 8 features with non-zero coefficients were finally obtained, as follows: original_firstorder_10Percentile, log.sigma.1.0.mm.3D_firstorder_90Percentile, log. sigma.2.0.mm.3D_glcm_Imc1, log.sigma.2.0.mm.3 D_ glcm_InverseVariance, log.sigma.4.0.mm.3 D_glcm _ InverseVariance, log.sigma.5.0.mm.3D_glcm_Inverse

Variance, wavelet.HLH_glcm_Idn, wavelet.HHL_ gldm_DependenceVariance, and wavelet.HHH_firstorder_ Median (Figure 4).

\section{AI model prediction results}

The above eight feature parameters were trained and tested for classification using LASSO to understand their ability to discriminate between COM and non-COM stones. The sensitivity, specificity and accuracy of the AI model were $90.5 \%, 84.3 \%$ and $88.5 \%$ for the training set, and
$90.1 \%, 84.3 \%$ and $88.3 \%$ for the testing set. The AUC of the training set was 0.935 [95\% confidence interval (CI): 0.907-0.962], the AUC of the testing set was 0.933 (95\% CI: $0.893-0.973)$, and the optimal cut-off value based on the training set was 0.470 (Figure 5).

\section{Discussion}

\section{Characteristics and treatment dilemma of COM stones}

Clinically, COM calculi are the most common, with an incidence almost twice that of COD calculi (18). Patients with urolithiasis have higher levels of COM in their urine than normal persons, thus causing greater damage to renal tubular epithelial cells. The damage to renal tubular epithelial cells is a prerequisite for the formation of renal tubular calculi. Damaged tubular epithelial cells can provide an effective site for stone core formation, while enhancing the adhesion of microcrystals to the cell membrane, accelerating stone formation (19).

A total of 543 patients with urinary calculi were included in this study, of which 373 were COM stones, accounting for $68.69 \%$ of all calculi. Ten cases were seen for recurrence, 7 after 1 year and 3 after 2 years; 5 cases recurred in the non-COM group, with AUA stones predominating. These data tentatively reflected the epidemiological characteristics of urinary stones in this region. COM calculi were more common, and the recurrence rate in this population was higher than that in non-COM calculi population, which was consistent with the epidemiological characteristics of stones reported in the literature (20). Other statistically significant features of COM included more male than female patients, with a male-to-female ratio of approximately $2.8: 1$, and 
Table 1 Clinical and imaging features of urinary calculi

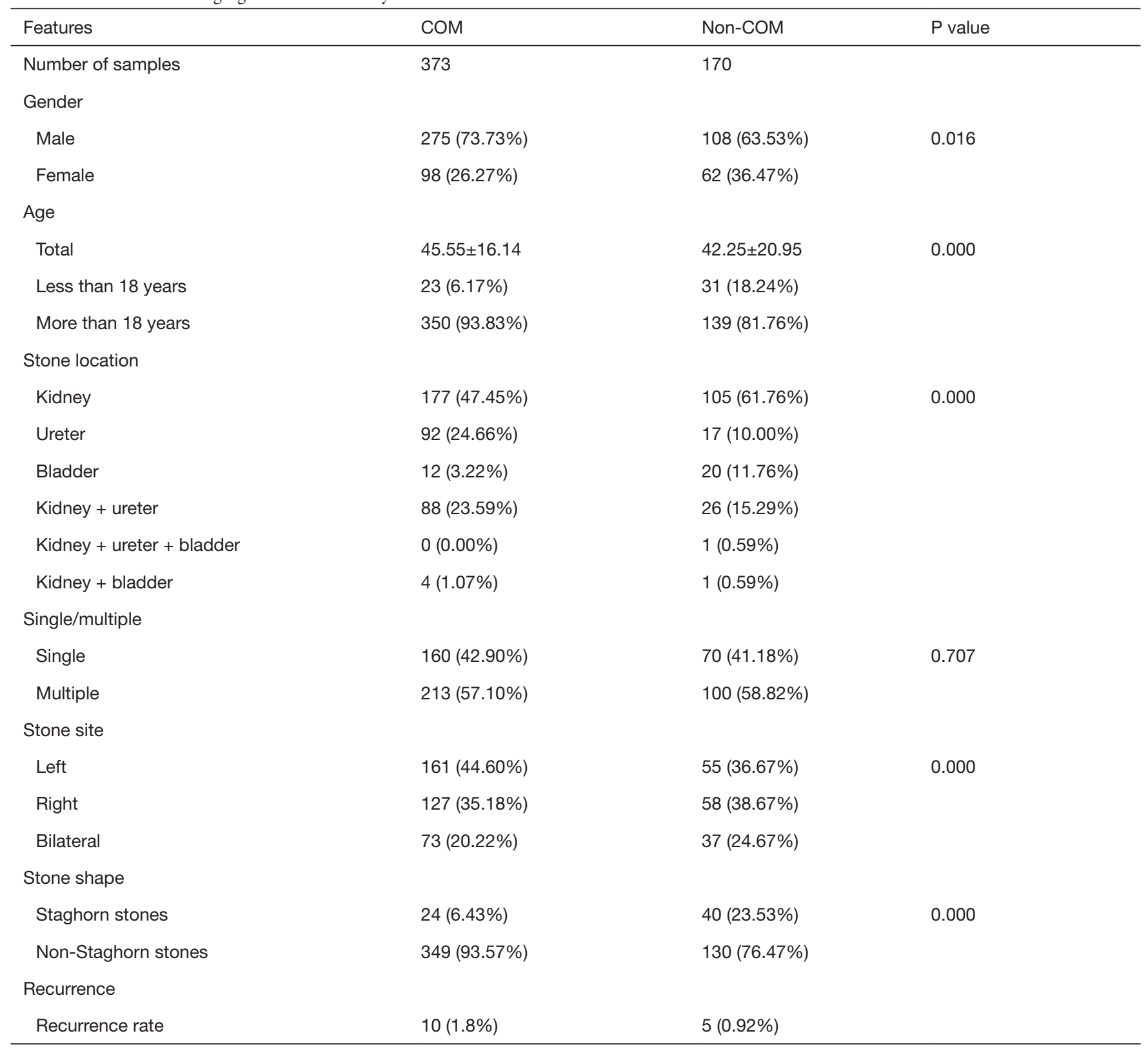

COM, calcium oxalate monohydrate.

more stones in adults aged $>18$ years, accounting for $93.83 \%$ of cases. The most common site was the kidneys $(177 / 373,47.75 \%)$, followed by the ureters $(92 / 373$, $24.46 \%)$, and the fewest occurred in the bladder (12/373, $3.22 \%)$. There were more stones on the left than right, and the fewest were bilateral. In terms of stone morphology, a small proportion $(24 / 373,6.31 \%)$ showed cast growth; among which, 3 cases were pure COM stones, 19 mixed stones, and the main mixed crystals were COD and/or CA. It can be seen that pure COM rarely forms staghorn stones, but when combined with other crystalline components, especially CA, it is more likely to grow in cast form.

The formation of COM is closely related to hyperoxaluria, and patients tend to have primary hyperoxaluria, intestinal disease, or long-term intake of foods with high oxalate load (21); therefore, the treatment 

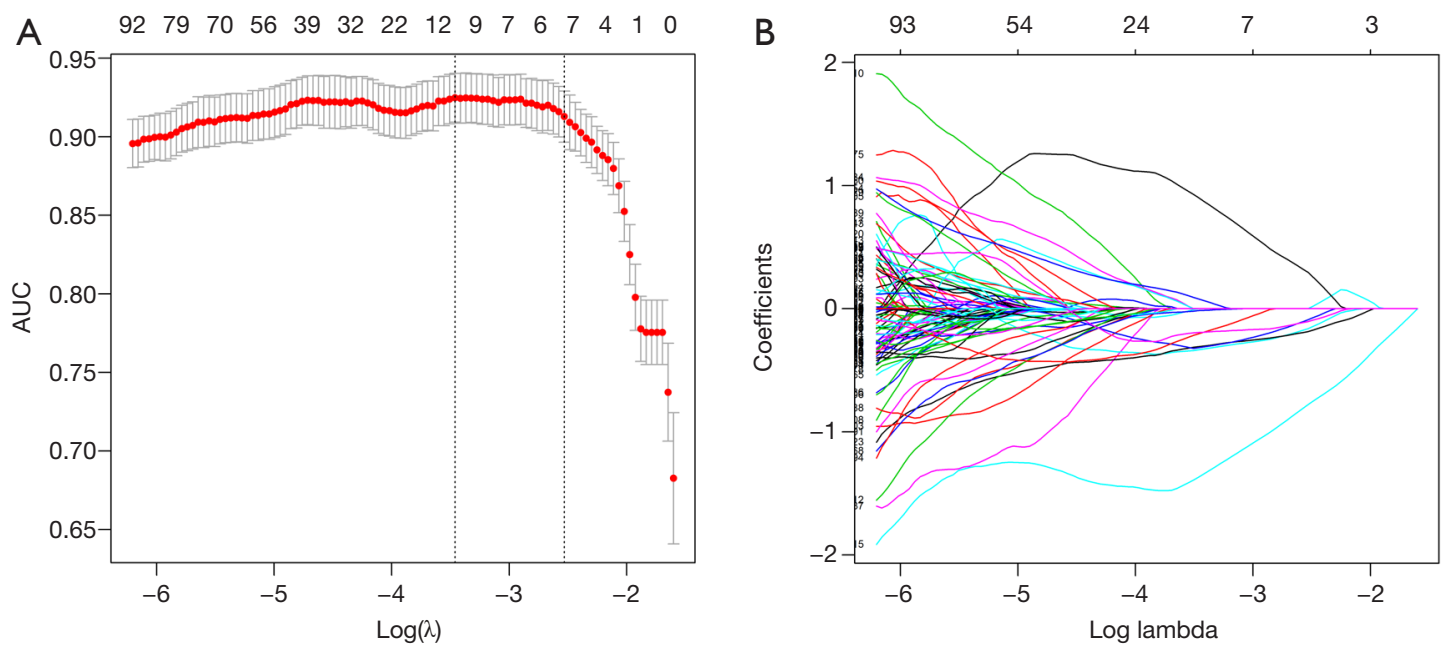

Figure 4 Screening of stone features.

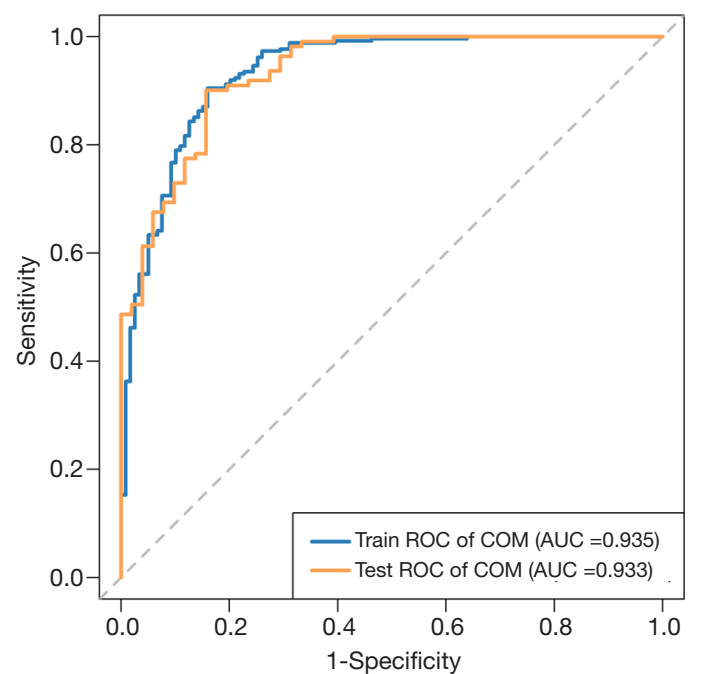

Figure 5 Performance of the calcium oxalate monohydrate (COM) model.

and prevention of these stones focuses on controlling hyperoxaluria. Important factors affecting the success rate of stone lithotripsy include the location, morphology, size, and composition of stones. Clinically, ESWL is often not ideal for hard, unbreakable stones, and patients often need a second operation, using flexible ureteroscope or PCNL. Therefore, for COM stones, which are the most common and difficult to fragment in clinical practice, identification of stone composition before surgery can help select the correct surgical method, reduce the risk of secondary surgery, avoid unnecessary injury, and reduce pain.

\section{Determination of stone composition with CT}

Unenhanced CT can accurately display the location, morphology and size of stones without interference from the intestine and surrounding tissues and organs, and the examination is easy and quick, which is the imaging test of choice for clinical diagnosis of acute renal colic and urinary tract stones (22). The density of stones varies depending on their chemical composition, and the range of attenuation values measured by CT also varies. Most of the previous studies have shown that CT can identify pure stones (23), but the predictive value for mixed stones is not certain.

The imaging principle of dual-energy CT (DECT) is that tissues with different atomic numbers have different levels of CT attenuation at high and low levels of X-rays, and this feature can be used to distinguish substances with similar electron densities. There are many studies related to stone composition analysis by DECT. Stolzmann et al. compared the attenuation difference of 40 calculi at 80 and $140 \mathrm{kV}$, and confirmed that DECT could accurately predict uric acid (UA) and non-UA calculi in vitro (24). Ilyas et al. (25) analyzed 60 stones in 53 patients and found that the sensitivity and specificity of DECT to differentiate UA and non-UA stones were both $100 \%$, and $97.8 \%$ and $92.3 \%$ for CO and non-CO stones. Many studies have shown that DECT can distinguish UA and calcified stones in vivo, with an accuracy of up to $100 \%$, and has potential for detection of mixed stones $(26,27)$. However, compared with unenhanced CT, the cost of DECT equipment and examinations are more expensive, the clinical promotion is insufficient, and it cannot be carried out as an 
emergency program.

\section{Advances in AI-related research on urinary stones}

In recent years, AI combined with imaging has been widely used in diagnosis, treatment, tumor grading and staging, and prognostic prediction of urinary system diseases $(28,29)$, and many noteworthy advances have been made in the field of urinary calculi. Kriegshauser et al. (30) examined 38 extracorporeal stone specimens using energy spectral CT, constructing and validating models using five parametric algorithms (support vector machine, random forest, artificial neural network, plain Bayesian tree, and decision tree). The results showed that all five models had 100\% accuracy in the differential diagnosis of UA and non-UA stones, while the random forest model had the best ability to identify guano, cystine and $\mathrm{CO}$ stones, with a prediction accuracy of $88 \%$ for CO stones. Ferrero et al. used a semi-automated method to segment stones and introduced quantitative indicators describing morphology to predict stone fragility, and the results showed high predictive value of DECT number ratio, volume and morphological indicators (31). A recent study collected 200 stone specimens (116 pure and 84 mixed stones) and used DECT combined with machine learning algorithms to construct an AI model, which was considered to predict the main chemical composition of urinary stones, with the accuracy of $91.1 \%$ (16).

In this study, the most commonly used unenhanced urinary tract $\mathrm{CT}$ and $\mathrm{AI}$ technology were combined to construct a COM stone prediction model using the LASSO algorithm. The accuracy of COM prediction was $88.3 \%$, and the sensitivity and specificity of AI model were $90.1 \%$ and $84.3 \%$, respectively. The AUC of the testing set reached 0.933 , suggesting that the model could accurately distinguish COM from non-COM stones, which was basically consistent with the performance of the previously reported DECT model $(16,30)$.

\section{Limitations}

Our study has several limitations. Firstly, this was a singlecenter study and due to the differences in medical equipment and scanning parameters among hospitals, it is unclear how well the AI model will work when applied to other centers. In the next step of the study, data from multiple centers can be incorporated to set up external test sets to further test the diagnostic performance of AI models. Secondly, only the most common COM stones were modeled; other types of stones were not analyzed due to the small sample size, and it is expected that more types of stones would be detected after adding data from other centers. Thirdly, our hospital routinely uses $\mathrm{Bf} 40$ and $\mathrm{Br} 40$ reconstruction kernels on CT data, which have edge-enhancing features that may have affected the CT attenuation values of stones. Moreover, data standardization before ROI feature extraction may remove the information that is contained in the mean CT number of the stone, which is associated with stone type. Finally, all stones were segmented by a single radiologist, and the reliability and repeatability of manual segmentation has an impact on the accuracy and robustness of the AI model, and the next step could be to try using a fully automated segmentation approach.

\section{Conclusions}

Unenhanced urinary tract CT is widely used in clinical practice, which is simple, convenient and fast. Combined with $\mathrm{AI}$, it can realize preoperative prediction of COM stones in vivo, which has high sensitivity, specificity and accuracy. Further research on AI models and their application to multicenter prospective clinical trials can help surgeons develop appropriate treatment and prevention plans.

\section{Acknowledgments}

Funding: This study was supported by the National Natural Science Foundation of China (No. 82060314) and Guizhou Science and Technology Foundation Project (QKHPTRC[2019]5803, QKHZC[2018]2793 and QKHJC[2017]1109).

\section{Footnote}

Reporting Checklist: The authors have completed the TRIPOD reporting checklist. Available at https://dx.doi. org/10.21037/atm-21-965

Data Sharing Statement: Available at https://dx.doi. org/10.21037/atm-21-965

Peer Review File: Available at https://dx.doi.org/10.21037/ atm-21-965

Conflicts of Interest: All authors have completed the ICMJE uniform disclosure form (available at https://dx.doi. 
org/10.21037/atm-21-965). BS serves as an Associate Editor-in-Chief of Annals of Translational Medicine from Sep 2020 to Aug 2021. XZ reports grants from Guizhou Science and Technology Foundation Project (QKHJC[2017]1109) and National Natural Science Foundation Project (No. 82060314). Rongpin Wang reports grants from Guizhou Science and Technology Foundation Project (QKHPTRC[2019]5803). Guangheng Luo reports grant from Guizhou Science and Technology Foundation Project (QKHZC[2018]2793). The other authors have no conflicts of interest to declare.

Ethical Statement: The authors are accountable for all aspects of the work in ensuring that questions related to the accuracy or integrity of any part of the work are appropriately investigated and resolved. The study was conducted in accordance with the Declaration of Helsinki (as revised in 2013). The study was approved by institutional ethics board of Guizhou Provincial People's Hospital (No. [2020]184) and individual consent for this retrospective analysis was waived.

Open Access Statement: This is an Open Access article distributed in accordance with the Creative Commons Attribution-NonCommercial-NoDerivs 4.0 International License (CC BY-NC-ND 4.0), which permits the noncommercial replication and distribution of the article with the strict proviso that no changes or edits are made and the original work is properly cited (including links to both the formal publication through the relevant DOI and the license). See: https://creativecommons.org/licenses/by-nc-nd/4.0/.

\section{References}

1. Muslumanoglu AY, Binbay M, Yuruk E, et al. Updated epidemiologic study of urolithiasis in Turkey. I: Changing characteristics of urolithiasis. Urol Res 2011;39:309-14.

2. Tae BS, Balpukov U, Cho SY, et al. Eleven-year Cumulative Incidence and Estimated Lifetime Prevalence of Urolithiasis in Korea: a National Health Insurance Service-National Sample Cohort Based Study. J Korean Med Sci 2018;33:e13.

3. Liu Y, Chen Y, Liao B, et al. Epidemiology of urolithiasis in Asia. Asian J Urol 2018;5:205-14.

4. Tundo G, Khaleel S, Pais VM Jr, et al. Gender Equivalence in the Prevalence of Nephrolithiasis among Adults Younger than 50 Years in the United States. J Urol 2018;200:1273-7.
5. Hsi RS, Kabagambe EK, Shu X, et al. Race- and Sexrelated Differences in Nephrolithiasis Risk Among Blacks and Whites in the Southern Community Cohort Study. Urology 2018;118:36-42.

6. Finkielstein VA, Goldfarb DS, et al. Strategies for preventing calcium oxalate stones. CMAJ 2006;174:1407-9.

7. Conti C, Casati M, Colombo C, et al. Synthesis of calcium oxalate trihydrate: New data by vibrational spectroscopy and synchrotron X-ray diffraction. Spectrochim Acta A Mol Biomol Spectrosc 2015;150:721-30.

8. Schubert G. Stone analysis. Urol Res 2006;34:146-50.

9. Yi PH, Kim TK, Yu AC, et al. Can AI outperform a junior resident? Comparison of deep neural network to first-year radiology residents for identification of pneumothorax. Emerg Radiol 2020;27:367-75.

10. Liu H, Cao H, Song E, et al. A cascaded dual-pathway residual network for lung nodule segmentation in CT images. Phys Med 2019;63:112-21.

11. Lin F, Cui EM, Lei Y, et al. CT-based machine learning model to predict the Fuhrman nuclear grade of clear cell renal cell carcinoma. Abdom Radiol (NY) 2019;44:2528-34.

12. Emblem KE, Pinho MC, Zöllner FG, et al. A generic support vector machine model for preoperative glioma survival associations. Radiology 2015;275:228-34.

13. Thongprayoon C, Krambeck AE, Rule AD, et al. Determining the true burden of kidney stone disease. Nat Rev Nephrol 2020;16:736-46.

14. Zhang GM, Sun H, Xue HD, et al. Prospective prediction of the major component of urinary stone composition with dual-source dual-energy CT in vivo. Clin Radiol 2016;71:1178-83.

15. Cloutier J, Villa L, Traxer O, et al. Kidney stone analysis: "Give me your stone, I will tell you who you are!". World J Urol 2015;33:157-69.

16. Große Hokamp N, Lennartz S, Salem J, et al. Dose independent characterization of renal stones by means of dual energy computed tomography and machine learning: an ex-vivo study. Eur Radiol 2020;30:1397-404.

17. Jung SK, Kim TW, et al. New approach for the diagnosis of extractions with neural network machine learning. Am J Orthod Dentofacial Orthop 2016;149:127-33.

18. Sun XY, Ouyang JM, Gan QZ, et al. Renal Epithelial Cell Injury Induced by Calcium Oxalate Monohydrate Depends on their Structural Features: Size, Surface, and Crystalline Structure. J Biomed Nanotechnol 2016;12:2001-14.

19. Chen S, Gao X, Sun Y, et al. Analysis of HK-2 cells exposed to oxalate and calcium oxalate crystals: proteomic insights into the molecular mechanisms of renal injury and 
stone formation. Urol Res 2010;38:7-15.

20. Grases F, Costa-Bauzá A, Ramis M, et al. Recurrence of renal lithiasis. Scand J Urol Nephrol 2003;37:482-6.

21. Bamberger JN, Blum KA, Kan KM, et al. Clinical and Metabolic Correlates of Calcium Oxalate Stone Subtypes: Implications for Etiology and Management. J Endourol 2019;33:755-60.

22. Türk C, Petřík A, Sarica K, et al. EAU Guidelines on Diagnosis and Conservative Management of Urolithiasis. Eur Urol 2016;69:468-74.

23. Stewart G, Johnson L, Ganesh H, et al. Stone size limits the use of Hounsfield units for prediction of calcium oxalate stone composition. Urology 2015;85:292-5.

24. Stolzmann P, Scheffel H, Rentsch K, et al. Dual-energy computed tomography for the differentiation of uric acid stones: ex vivo performance evaluation. Urol Res 2008;36:133-8.

25. Ilyas M, Dev G, Gupta A, et al. Dual-energy computed tomography: A reliable and established tool for In vivo differentiation of uric acid from nonuric acid renal Stones. Niger Postgrad Med J 2018;25:52-9.

26. Rudenko V, Serova N, Kapanadze L, et al. Dual-Energy Computed Tomography for Stone Type Assessment: A Pilot Study of Dual-Energy Computed Tomography with

Cite this article as: Tang $\mathrm{L}$, Li W, Zeng $\mathrm{X}$, Wang R, Yang X, Luo G, Chen Q, Wang L, Song B. Value of artificial intelligence model based on unenhanced computed tomography of urinary tract for preoperative prediction of calcium oxalate monohydrate stones in vivo. Ann Transl Med 2021;9(14):1129. doi: 10.21037/atm-21-965
Five Indices. J Endourol 2020;34:893-9.

27. Rompsaithong U, Jongjitaree K, Korpraphong P, et al. Characterization of renal stone composition by using fast kilovoltage switching dual-energy computed tomography compared to laboratory stone analysis: a pilot study. Abdom Radiol (NY) 2019;44:1027-32.

28. Li Q, Liu YJ, Dong D, et al. Multiparametric MRI Radiomic Model for Preoperative Predicting WHO/ISUP Nuclear Grade of Clear Cell Renal Cell Carcinoma. J Magn Reson Imaging 2020;52:1557-66.

29. Ström P, Kartasalo K, Olsson H, et al. Artificial intelligence for diagnosis and grading of prostate cancer in biopsies: a population-based, diagnostic study. Lancet Oncol 2020;21:222-32.

30. Kriegshauser JS, Paden RG, He M, et al. Rapid kVswitching single-source dual-energy CT ex vivo renal calculi characterization using a multiparametric approach: refining parameters on an expanded dataset. Abdom Radiol (NY) 2018;43:1439-45.

31. Ferrero A, Montoya JC, Vaughan LE, et al. Quantitative Prediction of Stone Fragility From Routine Dual Energy CT: Ex vivo proof of Feasibility. Acad Radiol 2016;23:1545-52. 